\section{ECCOMAS}

\section{Proceedia}

UNCECOMP 2021

$4^{\text {th }}$ ECCOMAS Thematic Conference on Uncertainty Quantification in Computational Sciences and Engineering M. Papadrakakis, V. Papadopoulos, G. Stefanou (eds.) Streamed from Athens, Greece, 28 -30 June 2021

\title{
MACHINE LEARNING AIDED STOCHASTIC SLOPE STABILITY ANALYSIS
}

\author{
Zhanpeng Liu ${ }^{1}$, Di Wu ${ }^{2}$, Daichao Sheng ${ }^{3}$, Behzad Fatahi ${ }^{4}$, Hadi Khabbaz \\ 12345 University of Technology Sydney
}

School of Civil and Environmental Engineering, University of Technology Sydney, Sydney NSW 2007, Australia

\author{
e-mail: 1'zhanpeng.liu@student.uts.edu.au \\ 2di.wu-1@uts.edu.au \\ 3daichao.sheng@uts.edu.au \\ ${ }^{4}$ behzad.fatahi@uts.edu.au \\ hhadi.khabbaz@uts.edu.au
}

\begin{abstract}
This paper presents the study in the machine learning aided stochastic slope stability analysis through the finite element method. The probability of failure of a dam with cohesive slope has been investigated. The numerical model has been built by the finite element method. An advanced machine learning algorithm called Extreme Learning Machine (ELM) is adopted to establish the regression model. The applicability and effectiveness of the presented approach are compared by the Monte-Carlo simulation method.
\end{abstract}

Keywords: Slope stability analysis, machine learning, finite element method, experimental design. 


\section{INTRODUCTION}

Slope stability is one of the principal considerations in soil mechanics such as rainfall induced landslide analysis and strength limit design of dam embankment. A series of research can be found in the slope stability analysis [1-11]. Herein, D.V. Griffiths presented research in slope stability analysis by finite elements methods [1], followed by the probabilistic slope stability analysis [2,3]. Nonlinear behaviour includes the nonlinear failure criterion and the yielding criteria on the elasto-plasitc analysis are investigated [4,5]. Reliability analysis for slope stability is presented by J.T. Christian et al. [6] and J.M. Duncan [7]. C.C. Huang et al. have conducted research in 3D slope stability analysis $[8,9]$. Research shows, because of the uncertainty of the soil properties and the large-scale variation of the nature environment, the deterministic analysis is not always leading the results that reflect the real-world situation. Thus, the non-deterministic analysis is of favoured in such a design, especially the reliability analysis for the slope stability.

This study presents a machine learning aided stochastic slope analysis by using extreme learning machine (ELM). ELM is a single hidden layer forward neural network (SLFN) that originally proposed by G.B. Huang [11], based on the four-layered feedforward neural network versus three $[12,13]$. Figure 1 illustrates the architecture of the classical ELM algorithm. The performance of the regression and multiclass classification is further discussed in $[14,15]$. Researchers investigated on the improvements of the ELM algorithms and the effectiveness of its extension including bidirectional extreme learning machine (B-ELM) [16]; incremental extreme learning machine (I-ELM) [17-19]; on-line sequential extreme learning machine (OS-ELM) [20]; OS-ELM with kernel [21] and so on. G. Huang has summarised the current research work in ELM in a review article up to 2015 [22]. In this paper, a numerical investigation has been conducted for the dam embankment model demonstrated in Figure 2. The results are compared with the conventional Monte Carlo Simulation method to demonstrate the accuracy and the efficiency of the presented method.

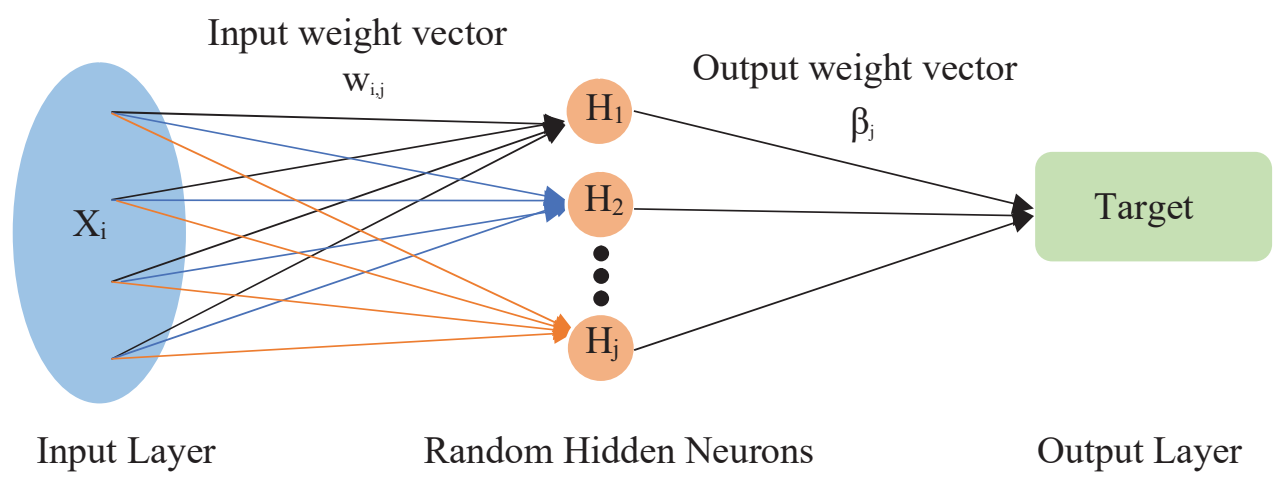

Figure 1: Classical ELM algorithm architecture 


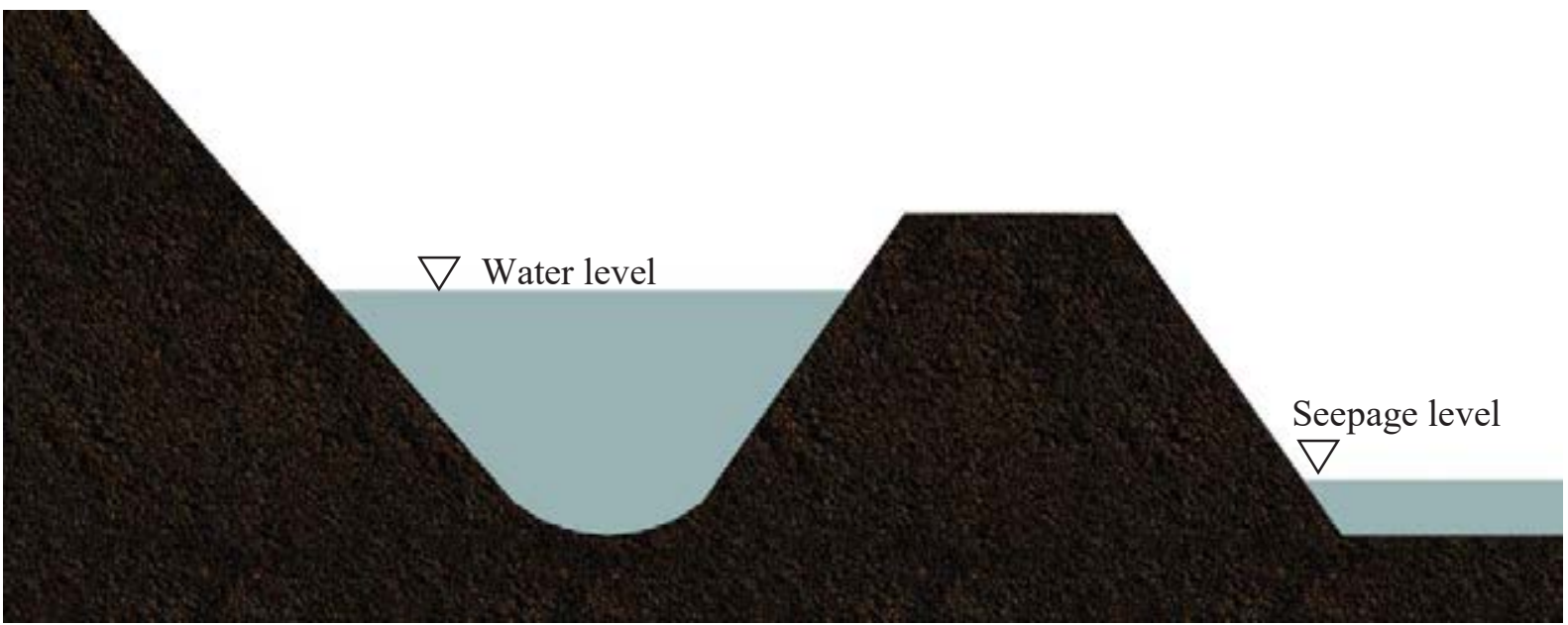

Figure 2: The illustration of the dam embankment

\section{PRELIMINARIES}

\subsection{Deterministic slope stability analysis by FE models}

The deterministic slope stability analysis has been widely investigated by finite element analysis [1-6], due to the page limitation, the fundamental of the finite element analysis will not be repeated. In this study, the slope stability analysis has been conducted by shear strength reduction method according to previous research [1-7]. The factor of safety (FOS) is used to reduce the cohesion thus results in a reduction of the soil shear strength; the FOS is formulated as:

$$
\mathrm{FOS}=\frac{c}{c^{\prime}}
$$

where $c$ is the material cohesion and $c^{\prime}$ is parameterised cohesion.

The Mohr-coulomb yield function is adopted, the associated plasticity can be demonstrated as:

$$
F=\frac{\sigma_{1}{ }^{\prime}+\sigma_{3}{ }^{\prime}}{2} \sin \left(\phi^{\prime}\right)-\frac{\sigma_{1}{ }^{\prime}-\sigma_{3}{ }^{\prime}}{2}-c^{\prime} \cos \left(\phi^{\prime}\right)
$$

where when $F>1$, the soil is yielding thus the stresses are redistributed. The failure of slope is determined in displacements, at a certain FOS value, the result does not converge due to the increment of reduction of the soil shear strength, which indicated the slope failure due to the instability.

\subsection{Reliability analysis through Extreme Learning Machine (ELM)}

In this section, the algorithm of the extreme learning machine is introduced. As a single hidden layer forward neural networks, the goal is to minimise the cost function:

$$
Y=\sum_{j=1}^{N}\left(\sum_{i=1}^{\widetilde{N}} \beta_{i} g\left(w_{i} \cdot x_{j}+b_{i}\right)-t_{j}\right)^{2}
$$

for $N$ samples and $\widetilde{N}$ hidden nodes. The input weight vector $w$ is randomly generated based on a continuous probability density function in an interval $[-1,1][11], b$ is the bias vector. The $g(x)$ is an activation function, in this study, Sigmoid function is adopted: 


$$
g(x)=\frac{1}{1+e^{-x}}
$$

The hidden layer output matrix $\mathbf{H}$ is formulated as:

$$
\mathbf{H}=\left[\begin{array}{ccc}
g\left(\mathbf{w}_{1} \cdot \mathbf{x}_{1}+b_{1}\right) & \cdots & g\left(\mathbf{w}_{\widetilde{N}} \cdot \mathbf{x}_{1}+b_{\widetilde{N}}\right) \\
\cdots & \ldots & \ldots \\
g\left(\mathbf{w}_{1} \cdot \mathbf{x}_{N}+b_{1}\right) & \cdots & g\left(\mathbf{w}_{\widetilde{N}} \cdot \mathbf{x}_{N}+b_{\widetilde{N}}\right)
\end{array}\right]
$$

leads to the output of ELM is given as follow:

$$
f(x)=\sum_{i=1}^{N} \beta_{i} h_{i}(x)=\mathbf{H} \beta
$$

where $\beta$ is known as the output weight vector that connects the hidden nodes and the output nodes, $\beta$ can be calculated by:

$$
\beta=\mathbf{H}^{\dagger} \mathbf{T}
$$

where $\mathbf{H}^{\dagger}$ is the Moore-Penrose generalized inverse of the hidden layer output matrix $\mathbf{H}$ [11].

\section{NUMERICAL INVESTIGATION}

For the purpose of demosntration, a numerical example is investigated by adoping the presented ELM algorithm and then compared with the Monte Carlo Simulation (MCS) method. In this example, the horizontal displacement and vetical displacement of a point $\mathrm{P}$ within the dam body are selected as targets for the ELM regression. The finite element model is illustrated in Figure 3. Soil properties are choosen to be random varibles, the Young's modulus, Poisson's ratio and soil porosity are uniformly distributed [1], where $E \sim U\left(0.995 \times 10^{5}, 1.005 \times 10^{5}\right) \mathrm{kPa}, v \sim U(0.2487,0.2512)$ and prosity $p \sim U(0.398$, 0.402); the soil density, soil cohesion and parameter $\tan (\phi)$ are lognormal distributed $[2,7]$, where $\rho_{\text {mean }}=2000 \mathrm{~kg} / \mathrm{m}^{3} \rho_{\text {sd }}=60 \mathrm{~kg} / \mathrm{m}^{3}, c_{\text {mean }}=10 \mathrm{kPa} c_{s d}=3 \mathrm{kPa}, \tan \left(\phi_{\text {sat }}\right)_{\text {mean }}=0.5774$ $\tan \left(\phi_{\text {sat }}\right)_{\text {sd }}=0.0115$, and $\tan \left(\phi_{\text {unsat }}\right)_{\text {mean }}=0.3640 \tan \left(\phi_{\text {unsat }}\right)_{\text {sd }}=0.0073 .100$ training samples are generated by latin hyper cube sampling method, computed by FEM and trained by ELM. In result verification, 10,000 samples are generated by the MCS method and computed by FEM. The results are compared between the trained ELM and MCS. The accuracy of the presented method is illustrated in Figure 4 and Figure 5. For the results presented in Figures 4 and 5, the total computional time of the MCS is over 200 hours, versus the presented ELM in 2 hours. It worth to mention that, FEM computation dominants the time consumption of ELM, by using the trained ELM, the testing time of newly generated 10,000 samples is within 1 second. In this example, following computer configurations are used:

System: Microsoft Windows 10

CPU: Intel Core i7 - 8665U $1.9 \mathrm{GHz}$

RAM: $16.0 \mathrm{~GB}$ 


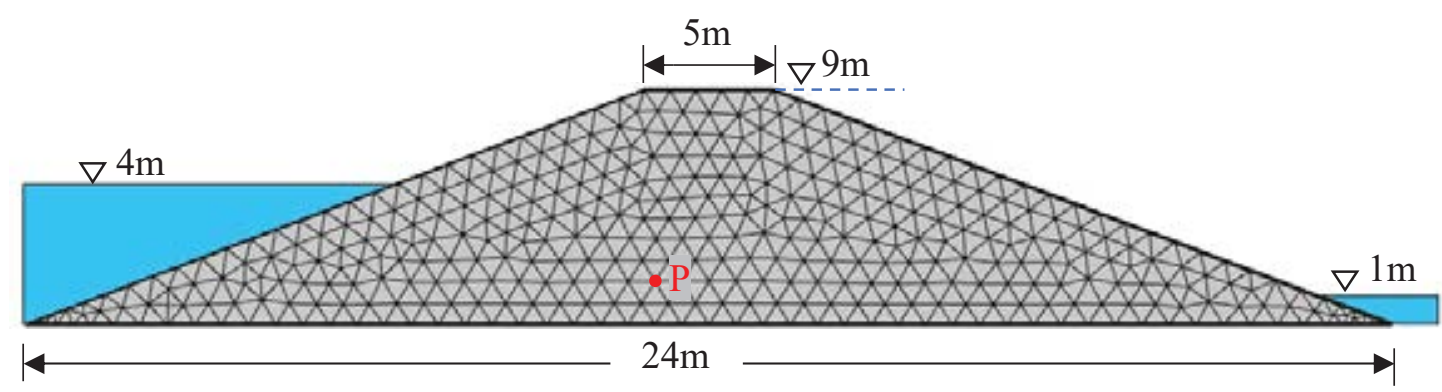

Figure 3: Numerical example in FEM
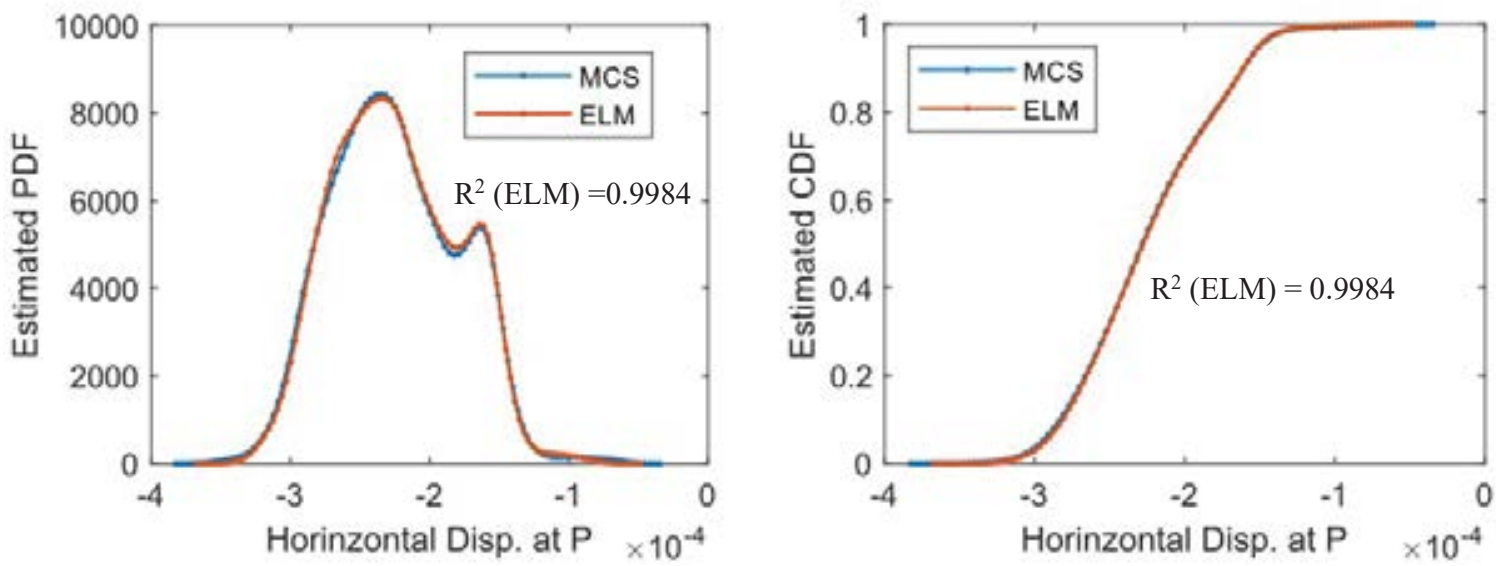

Figure 4: Horizontal displacement of the selected point. Unit: meter
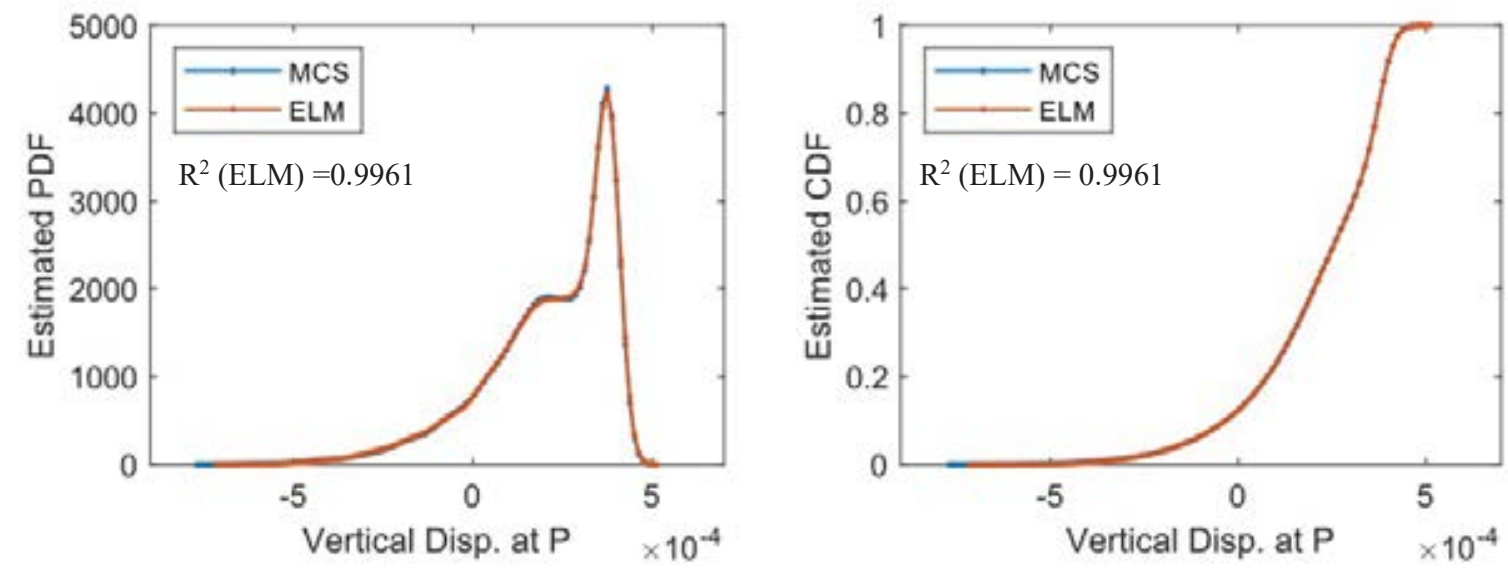

Figure 5: Vertical displacement of the selected point. Unit: meter

\section{CONCLUSIONS}

In this study, the machine learning aided stochastic slope stability analysis by extreme learning machine is conducted. Finite element method is adopted in the deterministic analysis. In order to verify the accuracy of the presented approaches, results are compared with Monte Carlo simulation. Results show the presented method agreed well with the Monte Carlo simulation while the computation efficiency is improved from 200 hours to 2 hours. Furthermore, the results have been illustrated and compared by probability density function and cumulative density function. 


\section{ACKNOWLEDGEMENT}

This research is supported by an Australian Government Research Training Program Scholarship.

\section{REFERENCES}

[1] D.V. Griffiths, P.A. Lane, Slope stability analysis by finite elements. Géotechnique, 49, No. 3, $387-403,1999$.

[2] D.V. Griffiths, G.A. Fenton, Probabilistic slope stability analysis by finite elements. Journal of Geotechnical and Geoenvironmental Engineering, 130:5, 2004

[3] D.V. Griffiths, J. Huang, G.A. Fenton. Probabilistic infinite slope analysis. Computers and Geotechnics. 38, 577-584, 2011

[4] X.L. Yang, J.H. Yin, Slope stability analysis with nonlinear failure criterion. Journal of Engineering Mechanics, 130(3), 267-273, 2004

[5] H. Zheng, D.F. Liu, C.G. Li, Slope stability analysis based on elasto-plastic finite element method. International Journal for Numerical Methods in Engineering, 64, 1871$1888,2005$.

[6] J.T. Christian, C.C. Ladd, G.B. Baecher, Reliability applied to slope stability analysis. Journal of Geotechnical Engineering, 120(12), 2180-2207, 1994.

[7] J.M. Duncan, Factors of safety and reliability in geotechnical engineering. Journal of Geotechnical and Geoenvironmental Engineering, 126(4), 307-316, 2000.

[8] G. Tang, J. Huang, D. Sheng, S.W. Sloan. Stability analysis of unsaturated soil slopes under random rainfall patterns. Engineering Geology, 245, 322-332, 2018.

[9] C.C. Huang, C.C. Tsai, Y.H. Chen, Generalized method for three-dimensional slope stability analysis. Journal of Geotechnical and Geoenvironmental Engineering, 128:10, 836-848, 2002.

[10] C.C. Huang, C.C. Tsai, New method for 3D and asymmetrical slope stability analysis. Journal of Geotechnical and Geoenvironmental Engineering, 126(10), 917-927, 2000.

[11] G.B. Huang, Q.Y. Zhu, C.K. Siew, Extreme learning machine: Theory and applications. Neurocomputing, 70, 489-501, 2006

[12] G.B. Huang, H.A. Babri, Upper Bounds on the Number of Hidden Neurons in Feedforward Networks with Arbitrary Bounded Nonlinear Activation Functions. IEEE Transactions on Neural Networks, Vol 9, No.1, 1998.

[13] S. Tamura, M. Tateishi. Capabilities of a four-layered feedforward neural network: four layers versus three. IEEE Transactions on Neural Networks, Vol. 8, No.2, 1997.

[14] G.B. Huang, H. Zhou, X. Ding, R. Zhang, Extreme Learning Machine for Regression and Multiclass Classification, IEEE Transactions on Systems, Man, And CyberneticsPart B: Cybernetics. Vol. 42, No. 2, 2012.

[15] G.B. Huang, C.K. Siew, Extreme learning machine: a new learning scheme of feedforward neural networks. International Joint Conference on Neural Networks (IJCNN2004), Budapest, Hungary, July 25-29, 2004 
[16] Y. Yang, Y. Wang, X. Yuan, Bidirectional extreme learning machine for regression problem and its learning effectiveness. IEEE Transactions on Neural Networks, Vol. 23, No.9, 2012.

[17] G.B. Huang, L. Chen, Convex incremental extreme learning machine. Neurocomputing, 70, 3056-3065, 2007.

[18] G.B. Huang, M.B. Li, L. Chen, C.K. Siew, Incremental extreme learning machine with fully complex hidden nodes. Neurocomputing, 71, 576-583, 2008.

[19] S. Song, M. Wang, Y. Lin. An improved algorithm for incremental extreme learning machine. Systems science \& control engineering, 8:1, 308-317, 2020.

[20] G.B. Huang, N.Y. Liang, H.J. Rong, P. Saratchandran, N. Sundararajan. On-line sequential extreme learning machine. the IASTED International Conference on Computational Intelligence (CI 2005), Calgary, Canada, July 4-6, 2005

[21] S. Scardapane, D. Comminiello, M. Scarpiniti, A. Uncini, Online sequential extreme learning machine with kernels. IEEE Transactions on Neural Networks and Learning Systems, Vol. 26 No. 9, 2015

[22] G. Huang, G.B. Huang, S. Song, K. You, Trends in extreme learning machines: A review. Neural Networks. 61, 32-48, 2015. 